\title{
COLONIALIDAD DEL SABER Y DOMINACIÓN EPISTEMOLÓGICA. APORTES ETNOGRÁFICOS PARA UN ANÁLISIS DE LA EDUCACIÓN INTERCULTURAL BILINGÜE EN EL ECUADOR DEL CORREÍSMO
}

\author{
COLONIALITY OF KNOWLEDGE AND EPISTEMOLOGICAL DOMINATION. \\ ETHNOGRAPHIC CONTRIBUTIONS FOR AN ANALYSIS OF INTERCULTURAL \\ BILINGUAL EDUCATION IN THE ECUADOR OF CORREISM
}

\author{
Marta Rodríguez-Cruz ${ }^{1,2}$
}

\begin{abstract}
El trabajo que presentamos tiene por objetivo analizar los mecanismos de reproducción de la colonialidad del saber y de la dominación de las epistemologías indígenas, a partir del análisis de los materiales e instrumentos de lectoescritura con los que se trabaja en diferentes escuelas andinas y amazónicas del sistema educativo ecuatoriano durante el correísmo. La metodología de investigación es cualitativa e integra técnicas etnográficas: análisis documental, observación participante y no participante y entrevistas semiestructuradas. Partiendo del reconocimiento de que la interculturalidad no es posible sin el "otro" y sin su descolonización, los hallazgos revelan que, con el nombre prostituido de interculturalidad, estos materiales e instrumentos sostienen el modelo educativo monocultural de matriz eurocéntrica dentro de la lógica de la colonialidad del saber. Los hallazgos también ponen de manifiesto que en este escenario algunos docentes indígenas son los que desarrollan prácticas educativas contestatarias mediante las que transmiten epistemologías indígenas, tratando de alterar la lógica de la colonialidad del saber.
\end{abstract}

Palabras claves: colonialidad del saber, dominación epistemológica, interculturalidad, pueblos indígenas

The objective of this work is to analyze the mechanisms of reproduction of the coloniality of knowledge and the domination of indigenous epistemologies, based on the analysis of the materials and reading-writing instruments with which students work in different Andean and Amazonian schools of the Ecuadorian educational system during the Correísmo. The research methodology is cualitative and integrates ethnographic techniques: documentary analysis, participant and non-participant observation and semi-structured interviews. Starting from the recognition that interculturality is not possible without the "other" and without its decolonization, the findings reveal that, under the prostituted name of interculturality, these materials and instruments support the monocultural educational model of Eurocentric matrix within the logic of the coloniality of knowledge. The findings also show that in this scenario, some indigenous teachers are the ones who develop answering educational practices through which they transmit indigenous epistemologies, trying to alter the logic of the coloniality of knowledge.

Key words: Coloniality of knowledge, epistemological domination, interculturality, indigenous peoples.

Sobre el papel, se han dado grandes pasos en el reconocimiento del derecho a una educación que respete y reproduzca la propia cultura y la propia lengua, o lo que es lo mismo, el derecho a la diferencia. Es el caso de Argentina, Colombia, México y Perú, reconocidos en sus cartas constitucionales como multiculturales y pluriétnicos o, yendo más allá, el de Bolivia y Ecuador, definidos en las mismas como interculturales y plurinacionales. Sin embargo, la incorporación de una terminología amable con la diversidad cultural en el discurso político-pedagógico, unida al reconocimiento de ese derecho a la diferencia, ha resultado ser uno más de los mecanismos por los que se reproduce la colonialidad del saber.

En el caso ecuatoriano, el régimen político que gobernó el país bajo el mandato de Rafael Correa (2007-2017), conocido como correísmo, trató de implementar el socialismo del siglo XXI, y, a través de la llamada Revolución Ciudadana, prometió descolonizar la ciudadanía y acabar con las relaciones de dominación gestadas sobre las categorías de raza y etnia. Una de las principales vías para tal fin fue la

\footnotetext{
1 Departamento de Antropología Social, Universidad de Sevilla. Programa Doctores PAIDI 2020, Fondo Social Europeo-Junta de Andalucía, Andalucía, España. marta.cruz.rodriguez@gmail.com

2 Departamento de Antropología, Historia y Humanidades FLACSO, Quito, Ecuador.
}

Recibido: octubre 2019. Aceptado: octubre 2020. 
de la educación, dirigida, al menos desde el discurso teórico-político, a la descolonización del saber y de las epistemologías de los pueblos y nacionalidades indígenas, dentro del nuevo modelo de Estado que desde 2008 se autodefinió como intercultural y plurinacional (Constitución Política, Art. 1, 2008). En consonancia, los niveles de enseñanza obligatorios -la Educación Inicial y General Básica- requirieron de una reconfiguración curricular que efectivamente condujera a esa descolonización del saber y a la consideración y puesta en valor de las epistemologías diversas, con el fin de construir a través de la educación esa nueva ciudadanía intercultural, plurinacional y descolonizada.

A partir de una etnografía desarrollada en distintas escuelas indígenas interculturales bilingües de la Sierra y Amazonía, el trabajo que presentamos tiene por objetivo analizar en qué medida durante el correísmo se ha logrado descolonizar el saber y las epistemologías indígenas en el sistema educativo -y concretamente en la Educación General Básica-, a través del examen de los materiales curriculares y extracurriculares y de otros instrumentos de lectoescritura. Para ello, en primer lugar, abordaremos el análisis de los debates teóricos y de las categorías conceptuales en torno al objeto de estudio. A continuación, explicaremos la metodología de investigación seguida para pasar al análisis y exposición de los resultados y, por último, cerraremos el trabajo con las conclusiones obtenidas.

\section{Colonialidad del Saber, Descolonización Epistemológica y Educación. Algunas Consideraciones Teóricas}

Numerosos expertos han abordado el estudio de la colonialidad, entendida como el desarrollo de procesos de dominación a escala mundial, liderados por Occidente a partir de la raza como principio estructurante. Este ejercicio de dominación toma forma a través de tres esferas fundamentales de la realidad: la del poder, la del ser y la del saber, cuya conjugación colonial ha dado lugar, respectivamente, a la colonialidad del poder, del ser y del saber (CastroGómez y Grosfoguel 2007; Escobar 2003; Grosfoguel 2011; Lander et al. 2000; Mignolo 1995, 2000, 2007, 2008; Quijano 2000).

Desde la estructura colonial hegemónica, la alteridad subalternizada, que en el Sur Global está integrada por la sociedad indígena y afrolatina, no conoce, no piensa y, por tanto, "no es". Sus saberes y pensamientos no tienen valor, lo que genera sentimientos de inferioridad en los oprimidos, que asumen como normales las prácticas, saberes y pensamientos hegemónicos y niegan los suyos propios (colonialidad del ser). A estas formas de colonialidad se suma la colonialidad del poder, basada en modelos hegemónicos de explotación y dominación configurados sobre el mismo principio de raza (y racismo) a partir del que se sitúa a la otredad también en una posición subalterna. Esto da cuenta del nacimiento desde el Sur Global de las teorías decoloniales (Castro-Gómez y Grosfoguel 2007; Escobar 2003; Grosfoguel 2011; Lander et al. 2000; Mignolo 1995, 2000, 2006; 2007, 2008; Quijano 1992, 2000), del planteamiento del giro decolonial (CastroGómez y Grosfoguel 2007; Césaire 2006 [1956]; Fanon 2010 [1952], 2003 [1961]; Maldonado 2008) y de la incorporación de la interculturalidad en el discurso de las luchas indígenas (Ferrão 2010; López 2009). Tres herramientas directamente dirigidas a la ruptura con las estructuras de dominación eurocentristas.

La colonialidad del saber -dimensión en la que nos centraremos en consonancia con el objetivo de este trabajo- está directamente relacionada con el racismo epistemológico. El mismo es entendido por Grosfoguel en su análisis dialógico sobre las teorías decoloniales de Fanon y Boaventura de Sousa Santos como aquella "jerarquía de dominación colonial en la que los conocimientos producidos por los sujetos occidentales" son considerados "superiores a los conocimientos producidos por los sujetos coloniales no-occidentales" (Grosfoguel 2011:102). Partiendo de esta base, debe señalarse la existencia, a su vez, de una superposición de jerarquías epistémicas dentro del mundo colonizado. Para el caso de América Latina, como lo refiere Aníbal Quijano (1992, 2000), existe una élite criolla, moderna, racista y, además, colonial. Se trata de una élite hegemónica blanco-mestiza colonizada por Occidente, que reproduce sobre sí misma y sobre la alteridad subalternizada los patrones de dominación mediante el ejercicio de la colonialidad del saber. Así, la colonialidad del saber -en íntima articulación con la del poder y la del ser- marca las relaciones asimétricas en las que las posiciones de poder epistemológico son siempre ocupadas por sectores blanco-mestizos, independientemente de que representen mayorías (casos de Ecuador y Perú) o minorías (como Bolivia y Guatemala), ya que el principio rector y estructurante que opera es la raza -y el racismo-. A consecuencia de ello, la alteridad subalternizada es sometida a un doble colonialismo epistemológico canalizado a través de los sistemas 
educativos: el externo -que viene de Occidente- y el interno -que viene de la sociedad nacional hegemónica blanco-mestiza y que favorece lo que González $(1963,2006)$ y Stavenhagen $(1963,1969)$ han dado en llamar colonialismo interno-.

Este colonialismo epistemológico insertado en escuelas y universidades tiene tres consecuencias fundamentales: la imposición de "esquemas teóricos pensados para realidades muy distintas" a la del "otro" del sujeto dominante (Grosfoguel 2011:102); el sometimiento de las epistemologías subalternas a una ceguera intencionada con la finalidad de que su visibilización no provoque la subversión del sistema de conocimiento dominante, de base eurocéntrica; $\mathrm{y}$, relacionado con lo anterior, la invisibilidad, y gracias a esta, la perpetuación de la dominación, explotación y violencia ejercidas sobre la alteridad subalternizada (Grosfoguel 2011:102). La elevación del conocimiento y la ciencia europeos como marco científico e intelectual dominante y, en consecuencia, la obliteración en los sistemas educativos de los conocimientos construidos por los pueblos indígenas perpetúa los modos de pensamiento coloniales (Maldonado 2007; Méndez 2012; Quilaqueo et al. 2014; Walsh 2008) y hacen urgente su descolonización epistemológica. Pues la comprensión del mundo es mucho más amplia que la comprensión occidental del mundo, y la diversidad, que incluye formas muy distintas de ser, pensar y sentir, es infinita (De Sousa Santos 2011).

En el caso de Ecuador, como en el de otros países del Sur Global, las epistemologías indígenas son tratadas por el sistema educativo como un conocimiento de segunda clase. Teñido de un pretendido ancestralismo folklorizante carente de cientificidad, es por ello minusvalorado por el conocimiento occidental, eurocéntrico y (auto) denominado científico, que invade los contenidos curriculares de las enseñanzas iniciales, básicas y medias. Sin embargo, el conocimiento eurocéntrico, que se ha presentado a sí mismo y al mundo como "descontaminado" de los saberes y elementos de otros sistemas culturales, en gran medida ha sido construido en base a elementos cognoscitivos propios de otras culturas árabes, americanas, asiáticas y orientales (Grosfoguel 2011; Pulido 2009), resultado del contacto colonial entre Occidente y los sistemas socioculturales de otras latitudes.

En la apuesta por la descolonización y aceptación del pluralismo epistemológico, el conocimiento occidental debe considerarse uno más entre otros posibles y, como tal, formar parte de un diálogo intercultural entre distintos sistemas de conocimiento desde un plano horizontal y, por tanto, no colonizante. Se trataría de lo que Boaventura de Sousa Santos (2007) ha denominado ecología de los saberes, para cuestionar la preeminencia y supremacía de un solo saber ante la multiplicidad de saberes existentes, ignorados e invisibilizados; de aquí que abogue por el pluralismo epistemológico.

Frente a la actual situación de colonialidad epistemológica en el sistema educativo ecuatoriano, la construcción de modelos pedagógicos desde un sentido de horizontalidad y reciprocidad sujeto-sujeto implicaría la construcción de una relación dialógica horizontal y recíproca entre sus epistemologías correspondientes, en beneficio de una interculturalidad epistémica decolonial. Esta se enmarcaría dentro de los postulados de Boaventura de Sousa Santos sobre la incompletud de las culturas y la hermenéutica diatópica (De Sousa Santos 2000), que pretende "ofrecer un procedimiento de traducción entre saberes pertenecientes a sistemas culturales diversos, así como un modelo para el diálogo intercultural" que permita "combatir el desperdicio de la experiencia a través del rescate de fragmentos culturales olvidados, marginados o descalificados (Vergalito 2009:20).

La descolonización epistemológica de la educación implica desconectarla del eurocentrismo (Quijano 1991) para desplazar el conocimiento occidental del centro de los modelos pedagógicos y ocuparlo con un pluriverso epistemológico. Se trata de girar epistemológicamente en la dirección de una educación verdaderamente integradora y no segregadora ni excluyente (Quilaqueo et al. 2014:274). Para esto es necesaria una rigurosa recuperación y sistematización de las epistemologías indígenas subalternizadas a fin de incorporarlas, verdaderamente, en las mallas curriculares oficiales. El objetivo fundamental del rescate, la incorporación, la visibilización y el conocimiento y reconocimiento de las epistemologías "otras" es la descolonización del sujeto colonizado y del sujeto colonizador, implicados en una misma lógica de dominación.

Para desarrollar un diálogo intercultural centrado en la descolonización epistemológica de la educación, cabe atender a la perspectiva crítica de la interculturalidad, desarrollada por autoras como Walsh (2010) para el contexto latinoamericano. Entendida como "proceso, proyecto y estrategia que intenta construir relaciones -de saber, ser, poder y de la vida misma- radicalmente distintas" (Walsh 2010:91), 
la interculturalidad crítica no se limita "a las esferas políticas, sociales y culturales” (Walsh 2010:89), al contrario, también se cruza con las del ser y el saber. Esto es, la interculturalidad crítica también se ocupa de "la exclusión, negación y subalternización ontológica y epistémico-cognitiva de los grupos y sujetos racializados por las prácticas -de deshumanización y subordinación de conocimientos- que privilegian a unos sobre otros, "naturalizando" la diferencia y ocultando las desigualdades que se estructuran y mantienen en su interior" (Walsh 2010:89).

En la misma línea, el líder aymara y sociólogo Simón Yampara señala la imposibilidad de construir una interculturalidad entendida en el sentido de Walsh $(2008,2010)$ si hay inequidad entre las culturas y desconocimiento del otro y de sus epistemes, lo que para los pueblos indígenas constituye "una especie de 'sublimación' de la colonización, puesto que no hay condiciones de equidad, menos de un diálogo de saberes y conocimientos que son presupuestos básicos para tejer un proceso de interculturalidad entre pueblos" (Yampara 2001:29).

Como señala De Sousa Santos (citado por Grosfoguel 2011:102), la diversidad epistemológica implica un diálogo de saberes que permita la incorporación de conocimientos y experiencias culturales no occidentales como punto de partida para retomar las experiencias desperdiciadas por la razón occidental". En el caso del sistema educativo ecuatoriano, y, dentro de este, del nivel de la Educación General Básica, nos preguntamos hasta qué punto ha existido un diálogo de saberes o si, por el contrario, ha seguido operando la colonialidad del saber durante el correísmo, bajo el discurso políticamente correcto de la interculturalidad.

\section{Metodología}

Los datos que presentamos fueron obtenidos a través de un trabajo de campo etnográfico desarrollado en un total de siete escuelas interculturales bilingües, durante 2013, 2014 y 2015, con una vuelta al campo en el 2018. Las escuelas se sitúan en comunidades indígenas y en ciudades de las regiones andina y amazónica, como se indica en la Tabla 1.

Todos los estudiantes se inscriben en alguna de las nacionalidades indígenas existentes en Ecuador. Los de las escuelas de la región andina pertenecen a la nacionalidad kichwa, mientras que, en la Amazonía, además de kichwas amazónicos, se registraron alumnos de las nacionalidades achuar, andoa, shiwiar, shuar, waorani y zápara. Los estudiantes tienen edades de entre cinco y 14 años y se inscriben en la etapa de la Educación General Básica -de primero a décimo grado-. El interés por esta etapa reside en su carácter de obligatoriedad, que, como tal, determina el tipo de educación que van a recibir los considerados "diferentes".

Aunque la etnografía abarcó numerosos aspectos del proceso educativo, en este trabajo abordaremos el análisis de tres elementos fundamentales: los contenidos curriculares de los libros escolares, los contenidos de las actividades realizadas al margen del currículum oficial y la lengua de lectoescritura en los procesos de enseñanza-aprendizaje. El abordaje analítico de dichos elementos corresponde al objetivo principal del trabajo que presentamos, relacionado con la colonialidad del saber.

Para el examen de los materiales curriculares se ha aplicado la técnica del análisis documental. Sin embargo, debe señalarse que durante el trabajo de campo encontramos limitaciones para acceder a los libros de texto, ya que son directamente distribuidos por el Estado a las escuelas y no pueden obtenerse en librerías. Ello nos obligó a solicitar los libros a los docentes al término de la jornada escolar diaria para recoger la información y devolverlos al día siguiente, con el fin de no obstaculizar el natural transcurso de los procesos educativos. En la mayoría de las ocasiones los datos tuvieron que registrarse mediante fotografía, ya que en gran parte de las comunidades no hay electricidad ni existen copiadoras ni escáner. A otros materiales curriculares complementarios -ejercicios preparados por los docentes- se accedió y fueron registrados en los mismos términos. Cabe, pues, advertir que la calidad de las imágenes presentadas responde a las duras condiciones en las que se desarrolló el trabajo de campo.

Junto a la técnica del análisis documental, también se aplicaron las de observación participante y no participante en los procesos de aula. Ambos tipos de observación siguieron un guion estructurado que estuvo orientado al registro de la enseñanza y el aprendizaje de los contenidos recogidos tanto en los libros escolares como en las actividades confeccionadas por los docentes, así como de la transmisión y uso de la lengua de lectoescritura en los procesos educativos. Adicionalmente, se aplicaron 20 entrevistas semiestructuradas a docentes y estudiantes y a algunos investigadores especializados en cosmovisiones y culturas indígenas. El análisis de la literatura especializada 
Tabla 1. Relación de instituciones educativas y ubicación geográfico-administrativa. List of educational institutions and geographic-administrative location.

\begin{tabular}{llllll}
\hline \multicolumn{1}{c}{ Escuela } & Comunidad & Ciudad & \multicolumn{1}{c}{ Parroquia } & Cantón & Provincia \\
\hline 1. Tránsito Amaguaña & ------- & Quito & La Argelia & Quito & Pichincha \\
\hline 2. José Ignacio Narváez & $\begin{array}{l}\text { San José de } \\
\text { la Bolsa }\end{array}$ & ------- & $\begin{array}{l}\text { Miguel Egas } \\
\text { Cabezas }\end{array}$ & Otavalo & Imbabura \\
\hline 3. Cacique Tumbalá & Zumbahua & -------- & Zumbahua & Pujilí & Cotopaxi \\
\hline 4. Pacha Quilotoa & Rumipamba & ------- & Guangaje & Pujilí & Cotopaxi \\
\hline 5. Amauta Nampi & --------- & Puyo & Shell & Pastaza & Pastaza \\
\hline 6. Sumak Yachana Wasi & Colimbuela & ------- & Imantag & Cotacachi & Imbabura \\
\hline 7. Centro Educativo & Bellavista & ------- & Canelos & Pastaza & Pastaza \\
Comunitario Río Villano & & & & &
\end{tabular}

sobre el objeto de estudio y la consulta de algunos artículos publicados en la prensa nacional también han constituido itinerarios metodológicos de esta investigación.

\section{Colonialidad del Saber e Invisibilidad Epistemológica. Mecanismos e Instrumentos de Reproducción Curriculares y Extracurriculares y Algunas Prácticas Contestatarias}

Antes de iniciar el desarrollo de este acá pite deben realizarse dos observaciones principales. La primera de ellas es la presentación y estructuración del conocimiento en asignaturas, a modo de compartimentos estancos desconectados entre sí, lo que contradice la lógica indígena en la que tal escisión no es posible; al contrario, las formas cognoscitivas y de explicación e interpretación de la realidad indígena son integrales y holísticas. La segunda de las observaciones tiene que ver con la concepción espacio-temporal lineal que estructura transversalmente los contenidos de los materiales escolares, frente a la concepción espaciotemporal cíclica del sistema de conocimiento indígena, ausente en los mismos ${ }^{1}$. Estos elementos representan, de partida, formas de colonialidad del saber.

Hechas estas primeras observaciones, pasamos ya al análisis propiamente dicho del tratamiento que reciben las epistemologías indígenas en los materiales escolares. En estos, el tratamiento subalterno de las mismas ha sido especialmente evidente en las materias de Matemáticas y Entorno Natural y Social, la presentación de los símbolos patrios y el uso de la lengua de lectoescritura en los procesos de enseñanzaaprendizaje, registrándose prácticas contestatarias a la colonialidad del saber en las actividades planteadas por docentes indígenas.

\section{Matemáticas}

En la materia de Matemáticas, el conocimiento descansa sobre matrices cognoscitivas occidentales, es presentado e ilustrado principalmente a través del universo simbólico, social y cultural propio de los modos de vida urbanos y blanco-mestizos y se transmite mediante herramientas de base eurocéntrica, como el diagrama de Gantt (Figura 1a) y las teselaciones (Figura 1b). Estas herramientas, que se explican en el libro de texto escolar, son puestas en práctica mediante la realización de los ejercicios correspondientes por parte del alumnado, como ha permitido constatar nuestra observación participante.

Una actividad frecuentemente observada en las materias de Matemáticas y Lengua y Literatura -sobre todo en los procesos de aula de los grados inferiores de la Educación Básica- es la de recitar entre todos los alumnos las tablas de multiplicar y el abecedario, respectivamente, bajo la dirección del docente. Asimismo, tanto en estas materias como en otras, además del empleo de la técnica del recitado y la memorización, se recurre frecuentemente al copiado de los contenidos de los libros de texto al cuaderno por parte del alumno. Nos llamó la atención que, junto a las anteriores, no se emplearan otras técnicas de enseñanza más adecuadas a las epistemologías y matrices cognoscitivas indígenas, como la Taptan ${ }^{2}$ para el caso de las Matemáticas. No obstante, debe anotarse que, si bien durante las fases de trabajo de campo desarrolladas en los años 2013, 2014 y 2015 ninguna de las instituciones educativas empleaba la Taptana, en el año 2017 el diario El Comercio publicó un artículo sobre etnomatemática en el que se afirma que la escuela Tránsito Amaguaña ya emplea este instrumento en sus procesos de enseñanza (Diario 


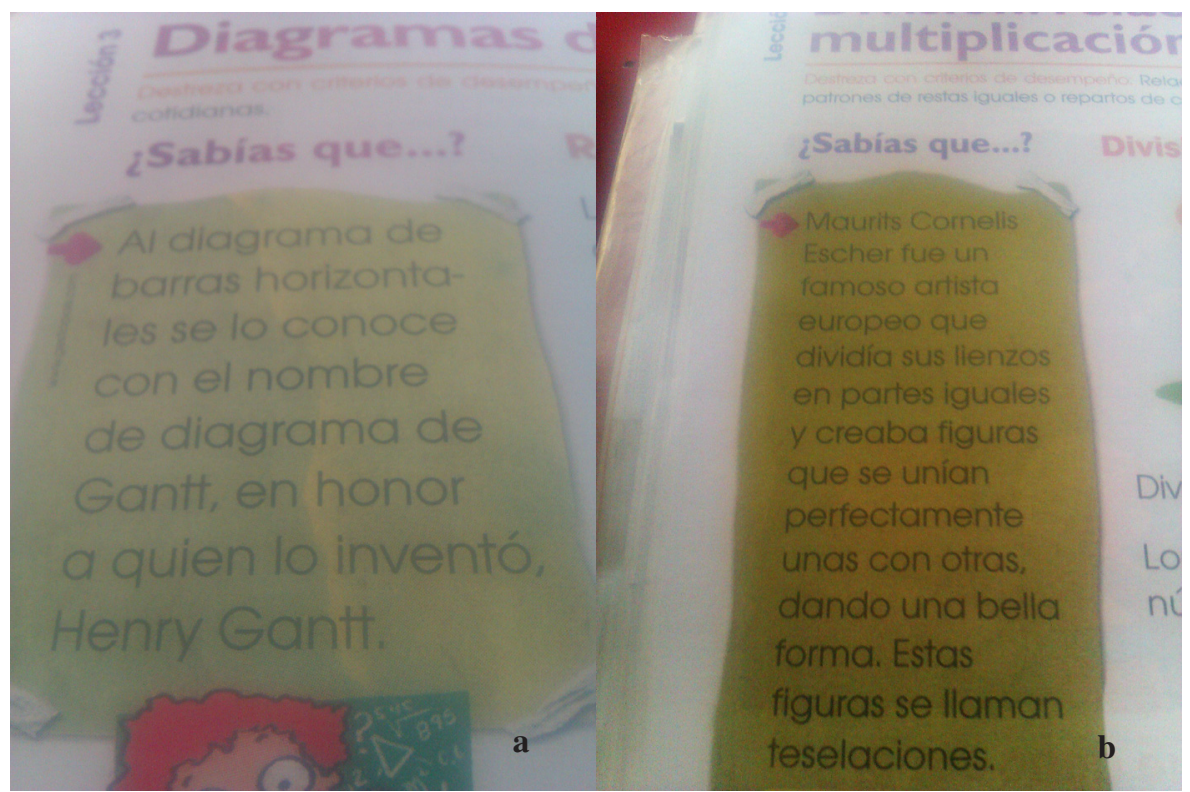

Figura 1. (a) y (b). Matemática. Cuarto de Educación General Básica. Libro del alumno. Ministerio de Educación-DINEIB.

(a) and (b) Mathematics. Fourth year of General Basic Education. Student's book. Ministry of Education-DINEIB.

El Comercio 2017). Sin embargo, esta incorporación responde a la iniciativa propia de la directora indígena de la escuela y no a su integración formal en el currículum oficial del sistema educativo para la Educación Básica, lo que no favorece el diálogo de saberes inscritos en los distintos universos epistemológicos.

De acuerdo con las investigaciones realizadas desde la etnomatemática, este instrumento milenario de cálculo mediante el que se pueden realizar grandes operaciones de suma, resta, multiplicación y división (Alquinga 2018; Castro 2014; Jurado 1993; Montaluisa 2006, 2010; Quizhpi 2019), y a diferencia del método tradicional, no se basa en la memorización sino en "el razonamiento lógico, la activación del pensamiento y la verificación concreta de los resultados" (Alquinga 2018:124).

Asimismo, tanto las investigaciones de Alquinga (2018) como las de Castro (2014) defienden la importancia de la Taptana desde el punto de vista cultural, identitario y pedagógico. Desde un punto de vista cultural e identitario la Taptana permite, mediante su incorporación en los procesos de enseñanza-aprendizaje, la construcción y solidificación de la identidad cultural y del sentido de pertenencia. Lo anterior es debido a que el concepto de número dentro de las cosmovisiones indígenas siempre se asocia a interpretaciones de los elementos del entorno que integran la realidad -como el número de integrantes de la familia, el número de granos de la cosecha, el número de productos que la familia saca al mercado, etc. (Quizhpi 2019)-. Por su parte, desde un punto de vista pedagógico, los resultados de las investigaciones han demostrado que esta herramienta permite "el desarrollo de la capacidad de concentración, rapidez mental y razonamiento matemático" (Castro 2014:4). Otros beneficios constatados son el fomento del trabajo grupal -frente a la individualidad de las técnicas de aprendizaje matemáticas occidentales- y un mejoramiento y fortalecimiento de la autoestima gracias a la identificación con la propia cultura (Castro 2014:5).

\section{Entorno Natural y Social}

En el caso de la materia de Entorno Natural y Social, como en las demás, la construcción del conocimiento sigue la misma tendencia occidental y su presentación igualmente responde de manera preponderante a matrices cognoscitivas eurocéntricas y a modelos y universos socioculturales blanco-mestizos, alejados del universo indígena, sobre todo del que se vive y reproduce en las comunidades. Por poner un ejemplo, en la unidad didáctica dedicada al estudio de los tipos de familia, los modelos familiares que se presentan son principalmente blanco-mestizos (Figura 2a). 


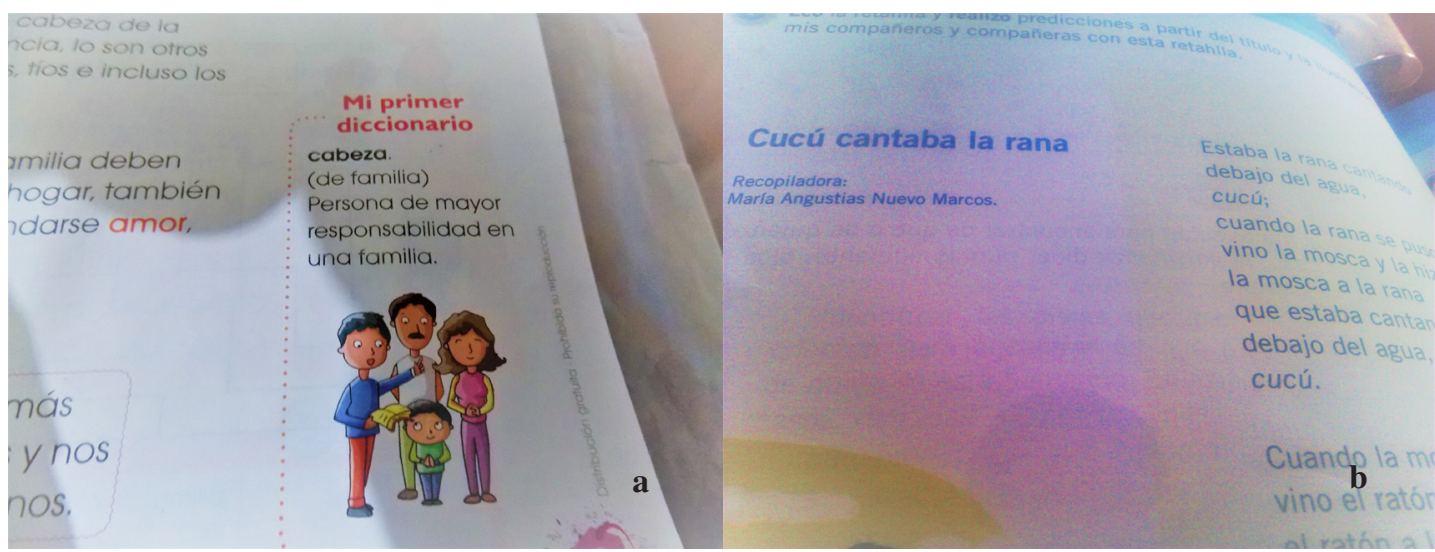

Figura 2. (a). Entorno Natural. Segundo de Educación General Básica. Libro del alumno, Ministerio de Educación-DINEIB. (b) Entorno Natural y Social. Cuarto de Educación General Básica. Libro del alumno, Ministerio de Educación - DINEIB.

(a) Natural Sciences. Second year of General Basic Education. Student's book, Ministry of Education-DINEIB. (b) Natural andSocial Environment. Fourth year of General Basic Education. Student's book, Ministry of Education - DINEIB.

A lo anterior se añade el registro de canciones y cuentos típicamente occidentales para la transmisión de los conocimientos que integran la materia (Figura 2b), en contraste con la invisibilidad a la que se someten en los libros de texto los mitos, poemas y canciones indígenas que transmiten saberes milenarios sobre esta área de conocimiento y que no han sido recogidos dada su inexistencia.

La totalidad de los docentes entrevistados, y de manera particular los indígenas, subrayan que "es más desde el mundo mestizo lo que se dice en el libro..." (comunicación personal, L.M., 20 de agosto de 2013, Quito) y señalan la escasez de contenidos relativos al universo social, cultural y económico de las comunidades y de los pueblos y nacionalidades indígenas, tanto en la materia de Ciencias Naturales como en el resto. De hecho, una de nuestras informantes indígenas expresó de manera abierta que: "A nosotros ese conocimiento del libro no nos vale. Nosotros, ¿cómo enseñamos? Con el libro del Estado, pero ¿qué cosas aprenden los guaguas ${ }^{3}$ a escribir? Las canciones de los abuelos" (comunicación personal, A.N., 10 de septiembre de 2013, Quito). En este mismo sentido, en varios de los procesos de aula observados se realizaron registros en los que algunos docentes indígenas incorporaban mitos, leyendas y cuentos ancestrales. En estos se transmitían conocimientos sobre la relación ser humano-naturaleza, el nacimiento de algunas naciones indígenas -como la kichwa o la shuar- y la importancia de la familia.

Otros registros etnográficos recogieron salidas al campo bajo la dirección de algunos docentes indígenas, quienes buscaban mayor congruencia entre los contenidos de enseñanza y los mundos de vida de los estudiantes. En estas salidas, los conocimientos impartidos tenían que ver con la explicación de la flora y la fauna del entorno, así como de las actividades productivas comunitarias. Presentamos el registro recogido durante la observación de una clase de Entorno Naural y Social, donde la docente decide obviar la parte correspondiente del temario y sacar a sus alumnos a explicar la tierra de su entorno:

Estamos en clase de Entorno Natural
y Social. La maestra dice que hoy
vamos a ir al campo a examinar la
tierra. Nos vamos al campo. Los niños
se sientan en semicírculo y la maestra
describe las características del terreno.
Aporta información local sobre plantas
endémicas y tipo de producción agrícola
de la zona. Habla de cuál es la época
de siembra, qué tipos de frutos se
siembran. Ahora les habla de la época
de la cosecha y lo relaciona con el Inti
Raimy ${ }^{4}$. Explica la importancia que
tiene esto para los indígenas, en qué
consiste, qué significado tiene. Habla
de los abuelos.

Estos docentes sostienen en sus relatos la necesidad de realizar tales actividades porque:

no vienen cosas de ellos, de la realidad de las comunidades, de nuestros alumnos indígenas, ahí yo utilizo otros medios, o 
sea, para podernos adaptarnos. Pongamos un caso de Ciencias Naturales, de Estudios Sociales, de Matemáticas. Por ejemplo, en Matemáticas qué se va a hacer. Se ha ido a una parcela y ahí a contar, aunque sea piedras, utilizando algunas plantas, sí se ha sabido observar. Por ejemplo, el caso de Ciencias Naturales, por ejemplo, a ver las enfermedades de la producción misma, qué producción tenemos por acá... o sea, cuáles son nuestras cosas. También mejor se ha salido por ahí a observación, a visitar las plantas, los animalitos, a conversar con los campesinos... (comunicación personal, 29 de mayo de 2014, Cotopaxi).

\section{Símbolos patrios}

También debe ser destacada la fuerte presencia de los símbolos patrios en los libros de texto. En todos ellos se hacen constantes referencias a la patria y a la "creación" de la misma a modo de refundación de la nación sobre las bases de la interculturalidad y la plurinacionalidad, principios sobre los que se reconoce el Estado ecuatoriano en el artículo 1 de su Constitución (2008). Un ejemplo de ello es la ilustración recogida en el libro de Lengua de séptimo de Educación General Básica (Figura 3).

La presentación de los símbolos patrios en los textos escolares es reforzada por otros elementos registrados mediante la observación en las instituciones educativas y en sus aulas. Por ejemplo, la presencia de la bandera ecuatoriana, su escudo y sus colores, el himno nacional y el himno a la patria (Figura 4).

Los himnos se recogen en los anexos de los libros de Lengua y Literatura en castellano y kichwa -no se han registrado en otras lenguas-. Particularmente, el himno nacional tiene mucha importancia en el proceso educativo: se han registrado transcripciones en las aulas de todas las escuelas y todos los lunes se celebra el Minuto Cívico. Para la celebración de este acto, toda la comunidad educativa se reúne, normalmente en el patio de la escuela, con la finalidad de cantar

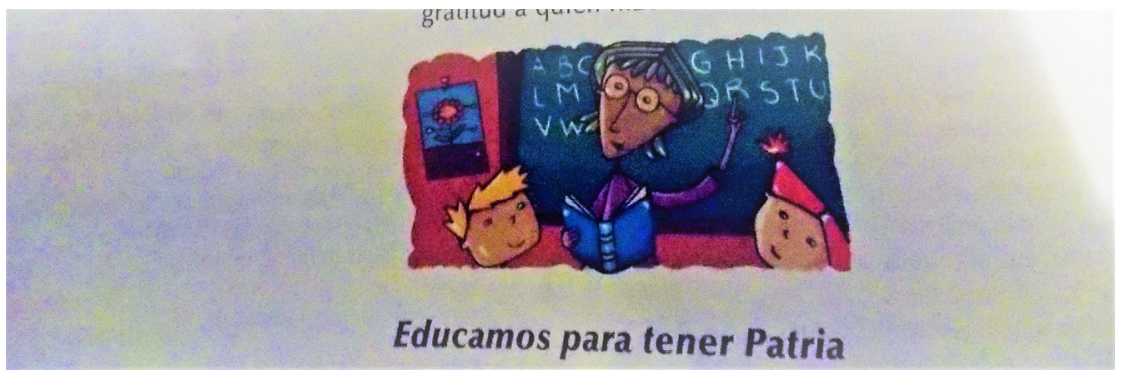

Figura 3. Lenguaje y Comunicación. Séptimo de Educación General Básica. Libro del alumno. Ministerio de Educación-DINEIB. Language and Communication. Seventh year of Basic General Education. Student's book. Ministry of Education-DINEIB.

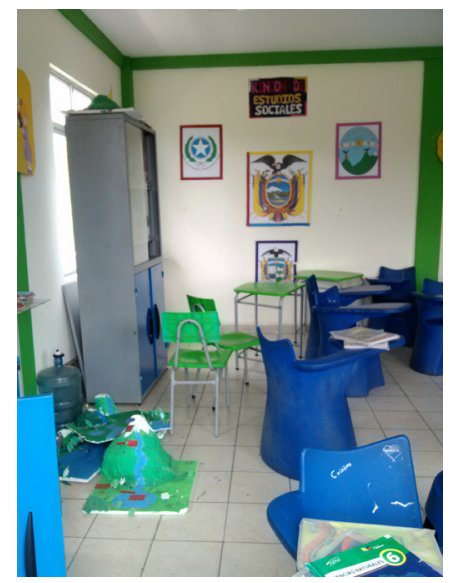

Figura 4. Aula de la escuela Sumak Yachana Wasi.

Sumak Yachana Wasi school classroom. el himno nacional y rendirle homenaje a la bandera de Ecuador. El acto es dirigido por un docente que ofrece indicaciones a los alumnos sobre cómo deben colocarse, qué gestos hacer -llevar la mano derecha al corazón, levantar la vista y mantenerla en la bandera-, cuándo empezar a cantar el himno y cuándo "romper filas". Todo el acto tiene un carácter patriótico, marcial y solemne (Figura 5).

Asimismo, las instrucciones para la celebración del Minuto Cívico vienen recogidas en el material escolar oficial, donde se presenta como ejercicio el de practicar "estas normas de respeto ante los símbolos patrios" (Figura 6). 


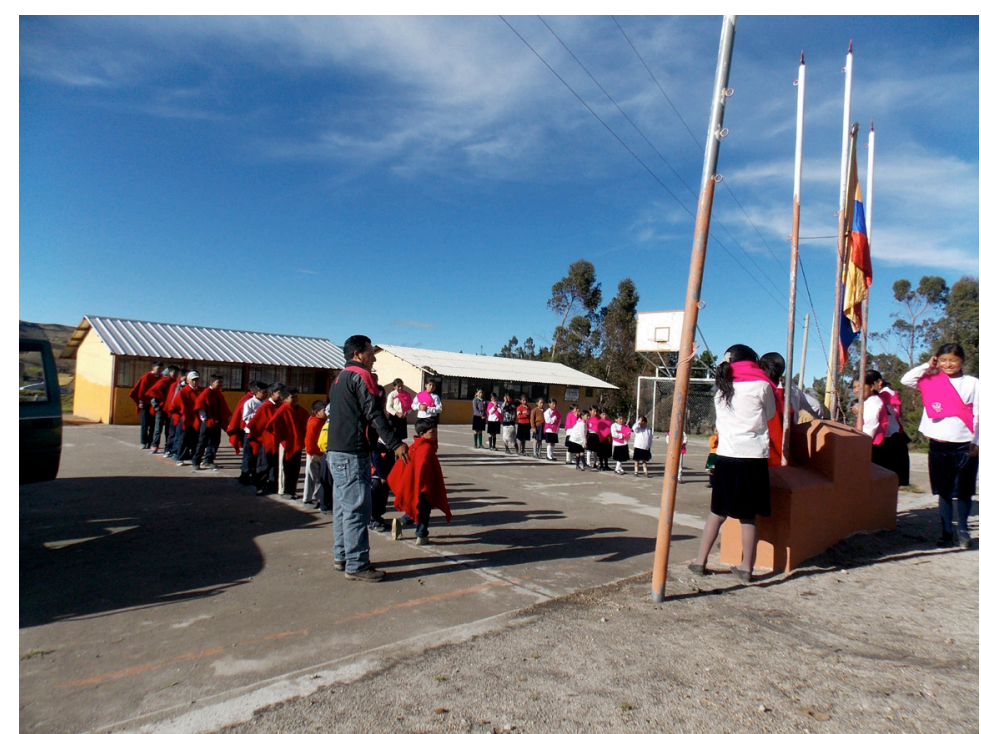

Figura 5. Celebración del Minuto Cívico en la escuela Pacha Quilotoa, mayo de 2014. Celebration of the Civic Minute at Pacha Quilotoa school, May 2014.

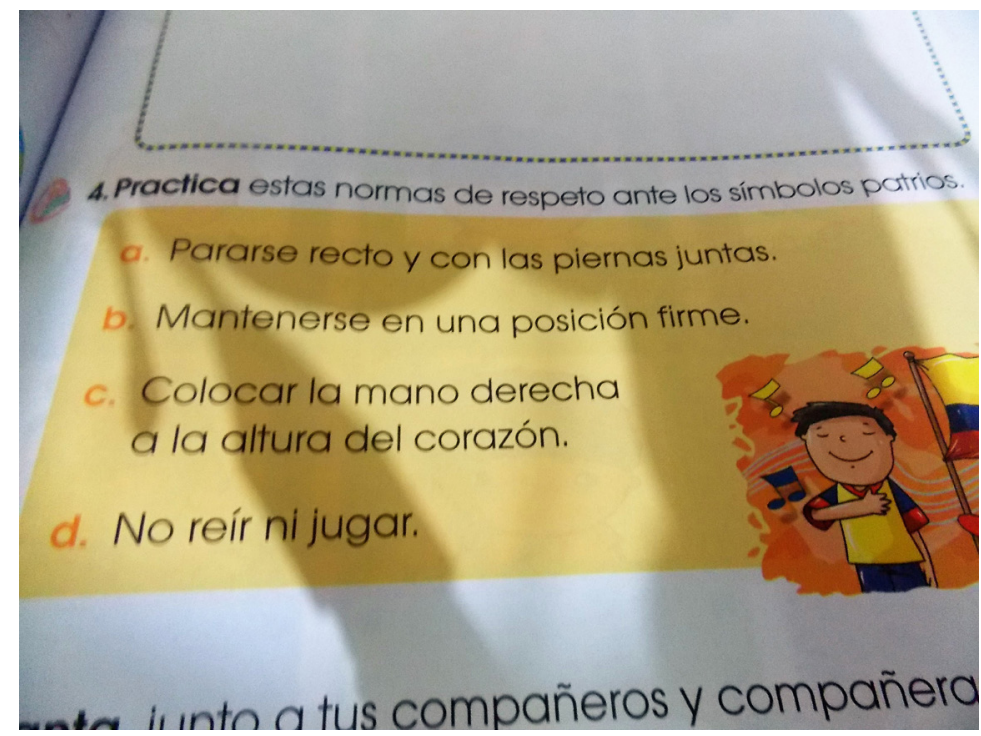

Figura 6. Entorno Natural. Segundo de Educación General Básica. Libro del alumno, Ministerio de Educación-DINEIB.

Natural Sciences. Second year of General Basic Education. Student's book, Ministry of Education-DINEIB.

En los libros escolares, los símbolos patrios no se ponen a dialogar con símbolos de los pueblos y nacionalidades indígenas tan importantes como la wiphala ${ }^{5}$, la cruz chakana prehispánica ${ }^{6}$ y las iconografías amazónicas hechas con el wituk ${ }^{7}$. Los registros revelan que estos elementos son incorporados en algunas actividades realizadas al margen del currículum oficial en dos de las siete escuelas que han formado parte de la investigación y que son dirigidas por mujeres indígenas: las escuelas Tránsito Amaguaña y Amauta Nampi. Solo en estas instituciones educativas se incorpora la wiphala para la celebración del Minuto Cívico y de otros actos institucionales. En el resto, los estudiantes indígenas incorporan las prácticas y símbolos de la sociedad dominante -en detrimento de los suyos propios-, como por ejemplo el himno y la bandera nacional para favorecer "situaciones de valores... la responsabilidad, la honestidad, etc. Pero 
no le hacemos nada a la wiphala" (comunicación personal, 14 de febrero de 2015, Imbabura).

\section{Lengua de lectoescritura}

Tanto el análisis documental de los libros escolares y de las actividades extracurriculares como la observación participante nos han permitido constatar que el conocimiento, en sus distintas áreas -Ciencias Sociales, Ciencias Naturales, Matemáticas, Lengua y Literatura-, se construye, difunde y reproduce en castellano, vehículo de transmisión de la episteme eurocéntrica y de sus correspondientes esquemas de pensamiento. Esto puede comprobarse en varias de las ilustraciones que hemos presentado a lo largo de estas páginas y que han sido directamente tomadas de los materiales con los que trabajan los estudiantes.

Asimismo, se ha constatado que en la asignatura de Lengua se estudia la historia del castellano, pero no se ha registrado ningún contenido dirigido al estudio de la historia de las 13 lenguas indígenas que existen en Ecuador. Para ilustrar la centralidad que adquiere el castellano en el proceso educativo, y particularmente, como lengua principal -y exclusivade transmisión del conocimiento, puede ponerse como ejemplo uno de los registros realizados en el libro de Lengua de séptimo de Educación Básica (Figura 7). Podrá notarse que el ejercicio se abre con el título "La vida de nuestro idioma", en alusión al castellano y evidenciando la línea excluyente entre lo "nuestro" -el castellano- y lo de "los otros" -las lenguas indígenas, que como parte de las epistemologías subalternas son efectivamente invisibilizadas-. En este sentido, y de acuerdo con los planteamientos de Albán (2010) y Veronelli (2016), podríamos hablar también de una colonialidad lingüística.

Lo anterior guarda una estrecha relación con el uso de la lengua de lectoescritura registrada en los procesos de aula mediante la observación. En estos, la lengua de interacción entre docente y alumnos es el castellano. Cuando el maestro es mestizo, esta es la única lengua de comunicación posible entre él y sus estudiantes, puesto que el docente no conoce la lengua materna de estos. En el caso de los docentes indígenas, la lengua de comunicación sigue siendo el castellano prácticamente en la totalidad de los casos estudiados. Solo si el maestro habla a sus alumnos de manera puntual en su lengua materna, ellos le contestan en el mismo idioma, aunque incluso en muchas ocasiones los alumnos siguen contestando en castellano. Esto, unido a la ausencia por motivos migratorios de referentes parentales y de otros en el ámbito doméstico que se encarguen de transmitir la lengua materna (Rodríguez-Cruz 2020), ha dado lugar al registro de situaciones en las que los estudiantes la desconocen:

"M.R.: ¿Sabes shuar?

A.R.: No...

M.R.: ¿Nada?

A.R.: No

M.R.: Pero tú eres shuar, ¿por qué no hablas tu lengua?

A.R.: No... no sé, solo sé español" (comunicación personal, 16 de abril de 2015, Pastaza)

Pese a la existencia de un Sistema de Educación Intercultural Bilingüe en el que la lengua indígena correspondiente debería ser la lengua principal de instrucción hablada y escrita, esta es soterrada en los procesos educativos en beneficio del castellano, dentro de la misma lógica de la colonialidad del saber. Teniendo en cuenta que el castellano es la lengua que se ocupa de la realidad de la sociedad dominante blanco-mestiza, las epistemologías que

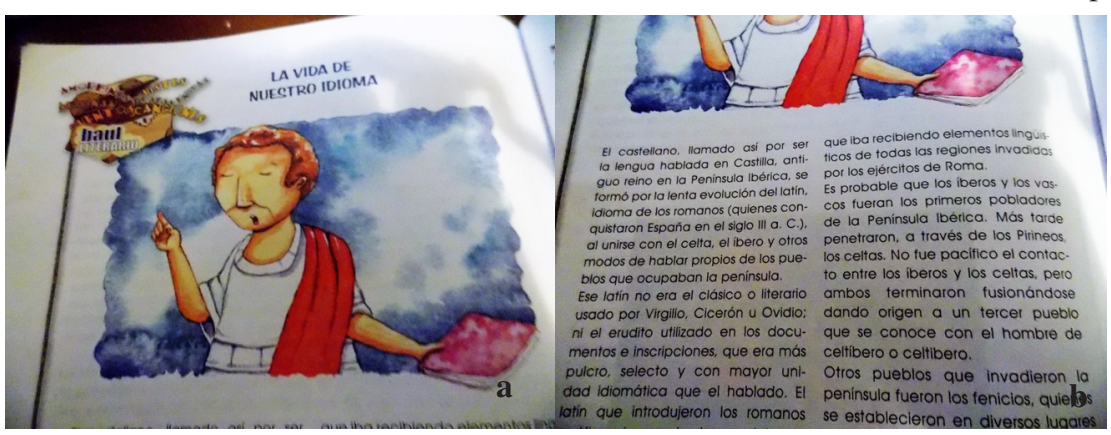

Figura 7. (a), (b). Lenguaje y Comunicación. Séptimo de Educación General Básica. Libro del alumno. Ministerio de Educación-DINEIB.

Language and Communication. Seventh year of Basic General Education. Student's book. Ministry of Education-DINEIB. 
se transmiten y reproducen a modo neocolonial son, correspondientemente, las blanco-mestizas en detrimento de las epistemologías y realidades propiamente indígenas.

Dicho de otra forma, el conocimiento está organizado en torno a centros de poder epistemológicos blanco-mestizos vehiculados por el castellano y periferias epistemológicas indígenas subalternizadas e invisibilizadas, cuyas lenguas vehiculares, equivalentemente, también lo están. Como lo señala Cuestas-Caza (2018), la descolonización de los países latinoamericanos no ha sido total, puesto que persisten mecanismos coloniales ocultos y redefinidos, entre ellos, los epistemológicos. Esta colonización epistemológica persistente consiste en "clasificar cuáles conocimientos, y por tanto cuáles lenguas son válidas y cuáles no" (Cuestas-Caza 2018:8).

Aunque la preeminencia del castellano pudo corroborarse mediante el análisis documental y la observación participante, los propios docentes la referían en sus entrevistas, insistiendo en que "el libro es castellano, todo es castellano acá" (comunicación personal, D.E., 26 de mayo de 2014, Puyo). Al respecto, nos parece interesante destacar los planteamientos de Gunther Dietz (2012) sobre la estandarización de las llamadas "lenguas nacionales" por parte de los Estados que, todavía hoy, entienden el plurilingüismo indígena como un problema y continúan promoviendo esa estandarización con el objetivo de "monolingüizar al conjunto de la [...] sociedad "nacional" (Dietz 2012:148). La escuela "intercultural bilingüe" sigue siendo una de las herramientas estatales privilegiadas para mantener la reproducción de ese monolingüismo.

Ante el actual panorama de la educación, de las epistemologías y de las lenguas indígenas, coincidimos con Rivera y Lehm (2013) en que una de las estrategias más importantes para su descolonización es la de la oralidad. Las lenguas coloniales que entraron en Abya-Yala desbancaron a las lenguas indígenas como el quechua, el aymara y el náhuatl, basadas en una oralidad que fue desprestigiada por la forma escrita de aquellas (Garcés 2007). Sin embargo, como lo refiere Rivera y Lehm (2013), la historia oral es un recurso epistemológico que permite mostrar el colonialismo y posibilitar el conocimiento de la historia de las naciones, pueblos y comunidades indígenas. Al rescatar los relatos orales, "podemos aprehender los factores subjetivos de la experiencia histórica y las reflexiones y doctrinas que orientaron el accionar, tanto individual como colectivo de los protagonistas" (Rivera y Lehm 2013:21). Recuperar los relatos orales en lenguas maternas -en el marco de las cuales contendrían todo su sentido y significadopermitiría conservar formas propias de enseñanza y de transmisión del conocimiento, así como la sabiduría, la historia y la identidad. Incorporar la oralidad y los contenidos indígenas en el currículum oficial junto a otras formas de conocimiento favorecería una apertura hacia un diálogo de saberes que debilitara la colonialidad de los saberes "otros".

\section{Actividades complementarias al currículum oficial planteadas por docentes}

Respecto a las actividades realizadas al margen del currículum oficial, la Ley Orgánica de Educación Intercultural (LOEI, 2011) que regula el sistema educativo determina en su artículo 92 que en las escuelas en las que se inscriben estudiantes indígenas debe existir un nivel microcurricular adaptado a las especificidades geográficas, socioculturales y lingüísticas de cada pueblo y nacionalidad y de cada comunidad. Ello requiere de una adaptación adecuada de estos materiales a los marcos epistemológicos de los estudiantes a los que van dirigidos. Aquí entra en juego un eslabón fundamental en todo modelo educativo, máxime si se trata de un modelo que se presume intercultural y que, como tal, debería ser consustancial a la decolonialidad epistémica: el docente.

$\mathrm{Si}$ bien se ha registrado una mayor pertinencia sociocultural en los materiales extracurriculares diseñados por docentes indígenas, en muchos de los materiales elaborados por estos y en la totalidad de los confeccionados por docentes blanco-mestizos se ha constatado un sesgo eurocéntrico. Entre estos materiales extracurriculares se encuentran fichas y ejercicios dedicados a la lectura, escritura y coloreado de sus contenidos típicamente occidentales. Es el caso de las actividades que se presentan a continuación, planteadas por dos docentes de dos escuelas distintas para los alumnos de cuarto y de sexto de Educación General Básica -respectivamente- en la asignatura de Lengua y Literatura. Como puede apreciarse, se trata de cuentos occidentales -Los Tres Cerditos y Pinocho (Figura 8)-.

Ya sean mestizos o indígenas, debe tenerse en cuenta que la formación que han recibido estos docentes en sus respectivas escuelas y, en su caso, universidades, también se inscribe dentro de los parámetros de la colonialidad del saber. No hay que perder de vista 


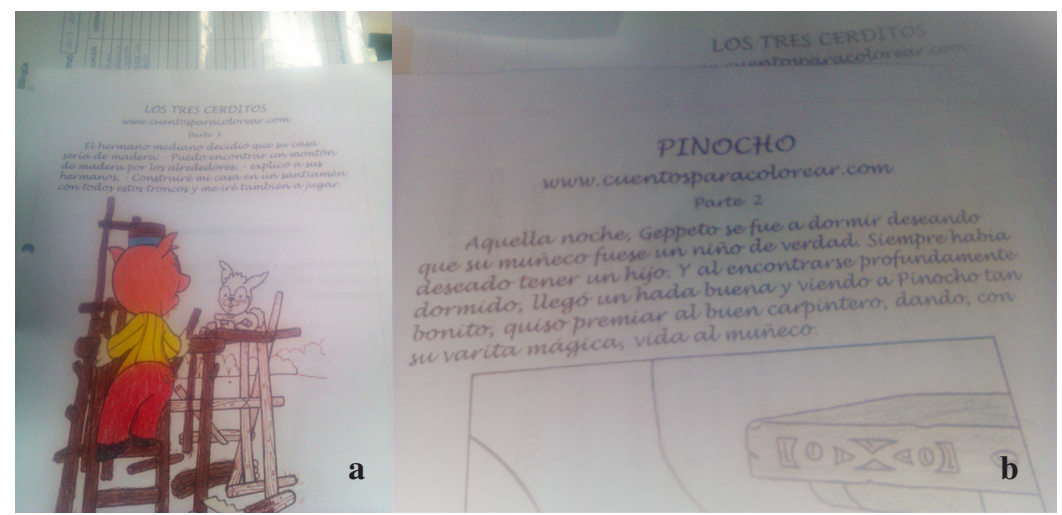

Figura 8. (a), (b) Materiales extracurriculares.

Extracurricular materials.

que, aunque desde posiciones distintas dentro de la jerarquía étnica establecida, docentes blanco-mestizos e indígenas forman parte de lo que Andrés Guerrero ha denominado estructura primaria de dominación (Burbano 1997). Dentro de la misma, la diferencia se instrumentaliza en términos de superioridadinferioridad, de manera tal que el inferior (indígena), el superior (blanco- mestizo) y las relaciones de dominación mantenidas entre ambos son construidas y sostenidas a partir de la diferencia étnica. Como consecuencia, los inferiorizados asimilan por mor de la dominación las epistemologías hegemónicas del grupo étnico dominante. En su esencia, como lo señala Guerrero, "la dominación está [...] vinculada con la matriz binaria de clasificación de las poblaciones" (Burbano 1997:64) a partir de la raza como principio estructurante, lo que, de nuevo, nos devuelve a la colonialidad. Es muy revelador el comentario que nos hace uno de los docentes cuando afirma que "la verdad nosotros recibimos una educación hispana [léase eurocéntrica], y peor yo soy mestizo y no conozco la cultura de ellos, no puedo enseñar otra cosa, pues" (comunicación personal, C.D., 12 de marzo de 2015, Imbabura).

Pero, como anticipábamos, si bien ha destacado la preeminencia de actividades de base eurocéntrica en los casos estudiados, en algunos de ellos sí se han registrado otras de mayor congruencia con las epistemes y mundos de vida indígenas. Cabe recalcar que los registros etnográficos apuntan a que cuando estas actividades adquieren mayor pertinencia, son siempre diseñadas por docentes indígenas, quienes fueron enculturados y socializados dentro de cosmovisiones indígenas y por ello poseen un mayor conocimiento de la cultura de los estudiantes y pueden realizar mejores adaptaciones de estas actividades.
No obstante, la lengua en la que se preparan estos ejercicios es en la práctica totalidad de los casos el castellano y no adoptan un enfoque en el que las distintas epistemologías se pongan a dialogar, dada la ausencia de formación en interculturalidad. Se presenta un ejemplo de una actividad propuesta por el maestro indígena de la unidad educativa Pacha Quilotoa para los alumnos de $3^{\circ}$ de Básica, recogido durante la observación:

Estamos en la asignatura de Entorno Natural y Social. El profesor no usa el libro de texto. Va a desarrollar una actividad con los alumnos para enseñarles cuál es la ubicación geográfica de su comunidad, qué otras comunidades hay, a qué cantón y a qué provincia pertenecen. La actividad la ha diseñado él. Dibuja en la pizarra lo siguiente y lo explica. Todo en castellano:

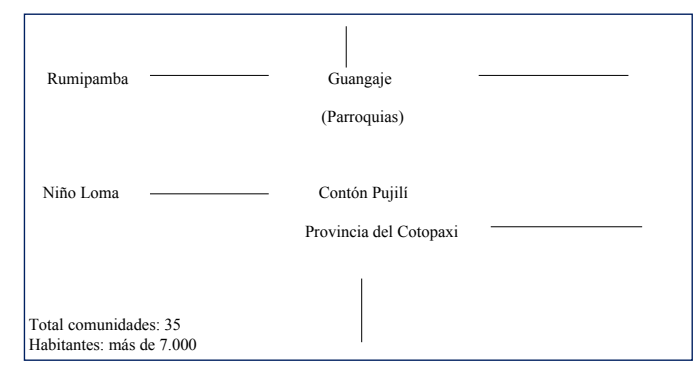

Realizando una interpretación libre de la idea de los "discursos ocultos" de James Scott (2000), podría afirmarse que estas prácticas desarrolladas "a espaldas" del sistema educativo son las que de alguna manera desafían la colonialidad del saber en las escuelas indígenas y alteran la lógica de la 
dominación a través del conocimiento. Lo anterior hace de estos docentes actores subversivos del orden epistémico dominante, sobre el que abren grietas en favor de la enseñanza de las epistemologías "otras".

\section{Conclusiones}

Los resultados obtenidos en esta investigación ponen de manifiesto que desde el ámbito educativo, y particularmente desde sus elementos constitutivos formales -como el currículum, los libros escolares y la lengua de lectoescritura-, no se promueve un diálogo de epistemologías plurales, pese al discurso políticamente correcto de la interculturalidad empleado durante el correísmo. Al contrario, se constata una preeminencia de la epistemología eurocéntrica, mediante la que se transmite y reproduce el sistema de pensamiento hegemónico en las distintas áreas de conocimiento analizadas. Los contenidos y metodologías pedagógicos registrados en los materiales oficiales reproducen la colonialidad del saber y silencian e invisibilizan las epistemologías indígenas en las distintas materias, en las que junto a las occidentales, aquellas deberían incorporarse a través de un diálogo epistémico horizontal e intercultural: junto al diagrama de Gantt y las teselaciones, la taptana; junto al modelo familiar occidental, el modelo familiar indígena; junto a los cuentos occidentales, los mitos y leyendas indígenas, fuentes de una sabiduría milenaria; junto a los símbolos patrios, la wiphala, la cruz chakana y las iconografías amazónicas hechas con el wituk.

Dentro de esta colonialidad del saber se observa, paralelamente, una colonialidad lingüística sobre las lenguas maternas del estudiantado, que se materializa en el predominio del castellano, registrado tanto en los materiales escolares como en los procesos de aula e interaccionales observados. La colonialidad de los saberes y epistemologías indígenas es correlativa a la colonialidad de las lenguas en las que aquellos se inscriben, retroalimentándose así una misma lógica de dominación. Una verdadera interculturalidad epistémica contemplaría, junto a la enseñanza del castellano, la de la correspondiente lengua indígena, e incorporaría también la oralidad como metodología de enseñanza de estas lenguas y de las epistemologías respectivas.

No obstante lo anterior, la colonialidad epistémica es contestada por algunos docentes indígenas mediante prácticas y ejercicios dirigidos a la incorporación de los saberes, conocimientos y formas de enseñanza propios en el proceso educativo. El desarrollo de prácticas educativas que transmiten elementos cognoscitivos e identitarios propios de los sujetos a los que van dirigidos permite la descolonización de sus saberes, la congruencia con sus realidades y la reproducción de su identidad, aproximándose a lo que de verdad debe ser el derecho a una educación que respete la diferencia. Puede afirmarse que estos docentes son actores subversivos de la lógica de la dominación a través del conocimiento.

Sin embargo, se trata de docentes que no han recibido la formación adecuada en interculturalidad, por lo que, pese a su laudable labor y a la importancia de su actividad contestataria, no disponen de las herramientas necesarias para poner a dialogar interculturalmente los universos epistemológicos que entran en contacto en las instituciones escolares. Estas constituyen el espacio en el que debería promoverse, a partir de todos los elementos que integran los procesos educativos, el diálogo entre epistemologías diversas en favor de la construcción de una verdadera sociedad intercultural.

Agradecimientos: Agradezco al Ministerio de Educación del Gobierno de España por financiar el proyecto "Políticas educativas en un Estado intercultural y plurinacional: Ecuador. Teorías y realidades" (FPU12/01696) y a los colegas, compañeras y compañeros de GEISA (Grupo para el Estudio de las Identidades Socioculturales en Andalucía) de la Universidad de Sevilla y de FLACSO-Ecuador por los aportes y contribuciones. Un agradecimiento especial y sincero a los docentes y estudiantes de cada una de las escuelas que formaron parte del estudio y a los miembros de las comunidades indígenas por su amable colaboración. También agradezco a los evaluadores de este artículo, quienes con sus comentarios han permitido enriquecerlo.

\section{Referencias Citadas}

Albán, A. 2010. Racialización, violencia epistémica, colonialidad lingüística y re-existencia en el proyecto moderno/colonial Colombia. En Debates Sobre Ciudadanía y Políticas Raciales en las Américas Negras, editado por C. Mosquera, A. LaóMontes y C. Rodríguez, pp. 197-221. Universidad Nacional de Colombia, Bogotá.
Alquinga, M. 2018. La enseñanza-aprendizaje de la matemática a través de la taptana. Anales de la Universidad Central del Ecuador 1(376):113-128.

Asamblea Nacional de Ecuador 2011. Ley Orgánica de Educación Intercultural. Registro Oficial No 417 (3 junio 2019) https:// bit.ly/2AoElei 
Burbano de Lara, F. 1997. Conversación con Andrés Guerrero. "Se han roto las formas ventrílocuas de representación". ICONOS, Revista de Ciencias Sociales 3:60-66.

Castro, J. 2014. Taptana Cañari (Taptana Nikichik). Comunicación presentada en el XII Coloquio Regional de Matemáticas y II Simposio de Estadística, pp. 1-5, Universidad de Nariño, Pasto.

Castro-Gómez, S. y R. Grosfoguel 2007. Prólogo. Giro decolonial, teoría crítica y pensamiento heterárquico. En El Giro Decolonial. Reflexiones para una Diversidad Epistémica más allá del Capitalismo Global, editado por S. Castro-Gómez y R. Grosfoguel, pp. 127-167. Iesco/Pensar/Silglo del Hombre, Bogotá.

Césaire, A. 2006 [1956]. Discurso sobre el Colonialismo. Akal, Madrid.

Constitución Política de Ecuador 2008. (3 junio 2019) https:// bit.ly/1Q1APPp

Cuestas-Caza, J.A. 2018. Runashimi: (de)colonialidad, poder y resistencia. Revista Filosofía Afro-Indo-Americana 7 (31):1-11

De Sousa Santos, B. 2000. Crítica de la Razón Indolente: Contra el Desperdicio de la Experiencia. Desclée de Brouwer, Bilbao.

De Sousa Santos, B. 2007. Beyond abyssal thinking. From global lines to ecology of knowledges. Eurozine (20 abril 2020) https:// www.eurozine.com/beyond-abyssal-thinking/

De Sousa Santos, B. 2011. Epistemologías del sur. Utopía y Praxis Latinoamericana 16 (54):17-39.

Diario El Comercio. La etnomatemática, un campo del saber que se abre paso en las aulas (20 junio 2019). https://bit.ly/2Mavamp

Diario La Hora. La cruz guarda un significado para cada cultura (25 enero 2019). https://bit.ly/2nNwPGB

Dietz, G. 2012. Multiculturalismo, interculturalidad y diversidad en educación. Una aproximación antropológica. Fondo de Cultura Económica, México D.F.

Escobar, A. 2003. Mundos y conocimientos de otro modo: El programa de investigación de Modernidad/Colonialidad latinoamericano. Tabula Rasa 1:51-86.

Fanon, F. 2003 [1961]. Los Condenados de la Tierra. Fondo de Cultura Económica, México D.F.

Fanon, F. 2010 [1952]. Piel Negra, Máscara Blancas. Akal, Madrid.

Ferrão, V. M. 2010. Educación Intercultural en América Latina: distintas concepciones y tensiones actuales. Estudios Pedagógicos, 36 (2): 333-342.

Garcés, F. 2007. Las políticas del conocimiento y la colonialidad lingüística y epistémica. En El Giro Decolonial: Reflexiones para una Diversidad Epistémica más allá del Capitalismo Global, editado por S. Castro-Gómez y R. Grosfoguel, pp. 217-242. Iesco/Pensar/Siglo del Hombre, Bogotá.

González, P. 1963. Sociedad plural, colonialismo interno y desarrollo. América Latina 6 (3):15-32.

González, P. 2006. El colonialismo interno: una redefinición. En La Teoría Marxista hoy: Problemas y Perspectivas, compilado por A. Boron, J. Amadeo y S. González, pp. 409-434. CLACSO, Buenos Aires.
Grosfoguel, R. 2011. La descolonización del conocimiento: diálogo crítico entre la visión descolonial de Frantz Fanon y la sociología descolonial de Boaventura de Sousa Santos. En Formas-Otras: Saber, Nombrar, Narrar, Hacer, editado por A. Vianello y B. Mañé, pp. 97-108. CIDOB, Barcelona.

Jurado, C. 1993. Didáctica de la Matemática en la Educación Primaria Intercultural bilingüe. Abya-Yala, Quito.

Lander, E. (compil.) 2000. La Colonialidad del Saber: Eurocentrismo y Ciencias Sociales. Perspectivas Latinoamericanas. CLACSO, Buenos Aires.

López, L.E. 2009. Interculturalidad, educación y política en América Latina: perspectivas desde el Sur. Pistas para una investigación comprometida y dialogal. En Interculturalidad, educación y ciudadanía. Perspectivas latinoamericanas, editado por L.E. López, pp. 129-218. Plural Editores, La Paz.

Maldonado, N. 2007. Sobre la colonialidad del ser: contribuciones al desarrollo de un concepto. En El Giro Decolonial. Reflexiones para una Diversidad Epistémica más allá del Capitalismo Global, editado por S. Castro-Gómez y R. Grosfoguel, pp. 127-167. Iesco/Pensar/Silglo del Hombre, Bogotá.

Maldonado, N. 2008. La descolonización y el giro des-colonial. Tabula Rasa 9:61-72.

Méndez, J. 2012. Eurocentrismo y modernidad. Una mirada desde la filosofía latinoamericana y el pensamiento descolonial. Omnia 28 (3):49-65.

Mignolo, W. 1995. La razón postcolonial: herencias coloniales y teorías postcoloniales. Revista Chilena de Literatura 47:91-114.

Mignolo, W. 2000. Diferencia colonial y razón postoccidental. En La Restructuración de las Ciencias Sociales en América Latina, editado por S. Castro-Gómez, pp. 3-28. Instituto de Estudios Sociales y Culturales, Bogotá.

Mignolo, W. 2007. El pensamiento decolonial. Desprendimiento y apertura. Un manifiesto. En El Giro Decolonial. Reflexiones para una Diversidad Epistémica más allá del Capitalismo Global, editado por S. Castro-Gómez y R. Grosfoguel, pp. 127-167. Iesco/Pensar/Siglo del Hombre, Bogotá.

Mignolo, W. 2008. La opción descolonial. Letral 1:4-22.

Ministerio de Educación de Ecuador-DINEIB 2010. Matemática. Cuarto de Educación General Básica. Edinum, Quito.

Ministerio de Educación de Ecuador-DINEIB 2011. Entorno Natural y Social. Cuarto de Educación General Básica. Don Bosco, Quito.

Ministerio de Educación de Ecuador-DINEIB 2011. Yachaypak Kukayo. Quinto de Educación General Básica. Don Bosco, Quito.

Ministerio de Educación de Ecuador-DINEIB 2012. Lenguaje y Comunicación. Séptimo de Educación General Básica. Edinum, Quito.

Ministerio de Educación de Ecuador-DINEIB 2012. Kukayo de Historia y Geografía. Octavo de Educación General Básica. Don Bosco, Quito.

Montaluisa, L. 2006. Ñuqanchiq Yachai (Nuestra Ciencia). Gráficas Arboleda, Quito. 
Montaluisa, L. 2010. Taptana Montaluisa. Universidad Politécnica Salesiana, Quito.

Pulido, G. 2009. Violencia epistémica y descolonización del conocimiento. Sociocriticism 24 (1):173-201.

Quijano, A. 1992. Colonialidad y modernidad/racionalidad. En Los conquistados. 1942 y la Población Indígena de las Américas, compilado por H. Bonilla, pp. 437-448. FLACSO/Tercer Mundo/ Libri Mundi, Quito-Bogotá.

Quijano, A. 2000. Colonialidad del poder: Cultura y conocimiento en América Latina. En Capitalismo y Geopolítica del Conocimiento: El Eurocentrismo y la Filosofía de la Liberación en el Debate Intelectual Contemporáneo, compilado por W. Mignolo, pp. 117-131. Ediciones del Signo, Buenos Aires.

Quilaqueo, D., S. Quintriqueo, H. Torres y G. Muñoz 2014. Saberes educativos mapuches: aportes epistémicos para un enfoque de educación intercultural. Chungara Revista de Antropología Chilena 46 (2):271-283.

Quizhpi, M.V. 2019. La Taptana Cañari en la Enseñanza de los Sistemas de Numeración a los Estudiantes de Primero de Bachillerato General Unificado, de la Unidad Educativa Shungumarca. Tesis de Máster, Facultad de Educación, Universidad Internacional de La Rioja, Cañar.

Rivera, S. y Z. Lehm 2013. Lxs Artesanxs Libertarixs y la Ética del Trabajo. Tinta y Limón, Buenos Aires.

Rodríguez-Cruz, M. 2020. Familia migrante, escuela y comunidad en los Andes equinocciales: continuidades y cambios en la identidad cultural. ICONOS, Revista de Ciencias Sociales XXIV (68):191-210.

Scott, J. 2000. Los Dominados y el Arte de la Resistencia. Discursos Ocultos. Era, México D.F.

Stavenhagen, R. 1963. Clases, colonialismo y aculturación. América Latina 6 (4):63-104.

Stavenhagen, R. 1969. Las Clases Sociales en las Sociedades Agrarias. Siglo XXI, México D.F.

Vergalito, E. 2009. Acotaciones filosóficas a la "Hermenéutica Diatópica" de Boaventura de Sousa Santos. Impulso 19 (48):19-30.

Veronelli, G.A. 2016. Sobre la colonialidad del lenguaje. Universitas Humanística 81:33-58.

Walsh, C. 2008. Interculturalidad, plurinacionalidad y decolonialidad: las insurgencias político-epistémicas de refundar el Estado. Tabula Rasa, 9:131-152.

Walsh, C. 2010. Interculturalidad crítica y educación intercultural. En Construyendo Interculturalidad Crítica, editado por J. Viaña, L. Tapia y C. Walsh, pp. 75-96. Instituto Internacional de Integración, Convenio Andrés Bello, La Paz.

Yampara, S. 2001. ¿Interculturalidad o "sublimación” de la neocolonización? En La Encrucijada Culturas: Anuario COSUDE 2001, editado por Agencia Suiza para el Desarrollo y la Cooperación, pp. 27-30. COSUDE, La Paz.

Notas

1 Dentro de las cosmovisiones indígenas el tiempo y el espacio representan dos realidades simultáneas e indesligables que integran una misma unidad denominada pacha, sin principio ni fin (comunicación personal, I.A., 20 de agosto de 2018, Quito).

2 Instrumento de cálculo prehispánico encontrado en la Provincia del Cañar, Ecuador. Según Montaluisa (2006), "esta piedra contiene dos matrices cuadradas de tres filas y tres columnas y 10 agujeros", mediante los que se podían hace grandes cálculos empleando granos y piedras colocados en los casilleros y agujeros.
Niños.

4 Fiesta andina de terminación de la cosecha en el solsticio de verano.

5 Bandera de los pueblos y nacionalidades indígenas.

6 Según Amaru Xavier Guamán, la misma era empleada por los pueblos prehispánicos de la región como una brújula que registraba la rotación de la tierra y como indicador de las épocas de siembra y cosecha según las estaciones del año (La Hora 2018).

7 Fruto del árbol del mismo nombre con el que los indígenas amazónicos preparan la pintura para decorarse el rostro. 